\title{
Precision transfer printing of ultra-thin AlInGaN micron-size light-emitting diodes
}

\author{
A.J. Trindade ${ }^{1}$, D. Massoubre ${ }^{2}$, B. Guilhabert ${ }^{1}$, D. Zhu ${ }^{3}$, N. Laurand ${ }^{1}$, E. Gu ${ }^{1}$, I.M. Watson ${ }^{1}$, \\ C.J. Humphreys ${ }^{3}$, M.D. Dawson ${ }^{1}$ \\ ${ }^{1}$ Institute of Photonics, SUPA, University of Strathclyde, 106 Rottenrow, Glasgow G4 0NW, UK \\ ${ }^{2}$ now at Queensland Micro- and Nanotechnology Centre, Griffith University, 170 Kessels Road QLD 4111, Australia \\ ${ }^{3}$ Department of Materials Science and Metallurgy, University of Cambridge, Pembroke Street, Cambridge CB2 3QZ, UK \\ *antonio.trindade@strath.ac.uk
}

\begin{abstract}
We report the fabrication of a mechanically-flexible 16x16 array of thin-film, micron-size LEDs emitting at $480 \mathrm{~nm}$. Devices were transfer-printed onto a mechanically-flexible ITO backplane using a modified, high-precision (placement accuracy $\pm 25 \mathrm{~nm}$ ) assembly system.
\end{abstract}

Introduction: Mechanically-flexible optoelectronics has become a highly attractive research topic in recent years [1] in part because it brings additional device and system capabilities to applications formerly limited by static, rigid surfaces. One major strand of investigation in this field involves the controlled transfer of micro- and nano-structures of inorganic semiconductors that act as active elements onto mechanically-flexible films. In this way, flexible arrays of light-emitting diodes (LEDs) and related components can be formed with envisioned applications encompassing conformable, intelligent displays, biosensor/monitors and communication systems [1].

There are two key stages to consider for the fabrication of such a flexible photonic platform: (i) the design of the active semiconductor structures to be transferred (i.e. material technology, size and morphology) and (ii) the transfer printing step (i.e. system/techniques used for the assembly and the printing/placement resolution). Concerning (i), III-nitride direct bandgap semiconductors represent the technology of choice for solid-state lighting and their use as active elements in flexible photonics is extremely advantageous since they can in principle cover the entire visible spectrum. Device miniaturization is also crucial in order to minimize stiffness and strain in the semiconductor when on the flexible substrate [1]. In that regard, micron-size LED formats [2] (surface area $<200 \mathrm{x} 200 \mu \mathrm{m}^{2}$ ) are particularly well-suited especially if the active region thickness is made small (thin-film LEDs). For (ii), transfer-printing precision is important and will become even more so when fully nanoscale active elements are used. Some groups have reported device placing accuracies of $\sim 1$ to $2 \mu \mathrm{m}[3,4]$, but this precision will be inadequate when targeting lithography-limited features or when placing arrays of devices within close proximity. There is therefore a need to enable transfer printing of optimized thin-film semiconductor structures with higher assembly precision.

In this context, we report here the demonstration of the pick-and-place transfer printing of an AlInGaN LED array. The process was realized using a commercial nano-patterning system (Nanoink Inc. NLP2000) converted into a computer-controlled transfer-printer. The system is based on a 5-axis stage (3 linear stages and 2 goniometers) combined with high resolution optical encoders. As a result, a long traveling range covering a $100 \times 100 \mathrm{~mm}^{2}$ area can be achieved while maintaining an accuracy of as small as $\pm 25 \mathrm{~nm}$ with precise tip and tilt control. In addition, an extensive software suite with CAD allows the step-by-step control and automatization of transfer printing. LEDs grown on silicon (Si) substrates were used to fabricate a 16x16 array of thin micro-LEDs (total LED thickness of $2 \mu \mathrm{m}$ ). The thin-film devices thus fabricated have emission spectra centered at $480 \mathrm{~nm}$ in a complete mechanically-flexible configuration. We note that these represent, to our knowledge, the thinnest LED structures transfer-printed onto a flexible substrate.

Fabrication: AlInGaN micro-LED $\left(150 \times 150 \mu \mathrm{m}^{2}\right)$ arrays on Silicon (111) [5] were processed and transferred to an acceptor substrate. To help the release of devices, anisotropic wet etching $(\mathrm{KOH})$ was used to remove the AlGaN buffer layer and part of the Si substrate resulting in micro-LEDs suspended by two sacrificial anchors prior to the transfer step (Figure 1.a). From the epilayer design, the resulting device thickness is $\sim 2 \mu \mathrm{m}$ [6]. Elastomeric stamps for the pick-and-place transfer process of the LED structures were made of polydimethylsiloxane (PDMS) made by a molding process involving a silicon (100) wafer - etched to create pyramidal recesses - and cells of SU-8 epoxy to define the main body (Figure 1.b). The acceptor substrate is mechanically flexible and composed of a polyethylene terephthalate (PET) thick base layer coated with $100 \mathrm{~nm}$ of indium tin oxide (ITO), which was 
patterned as an interconnecting electrical circuit for the transferred devices (Figure 1.c). Final device assembly was performed with the previously described nano-positioning system. Each transfer-printed micro-LED was connected to the PET/ITO acceptor substrate using precisely located droplets of conductive epoxy.

Experimental results: Figure 2 is a schematic describing the transfer process. Elastomeric stamps are precisely placed to match each device and compressed. Due to viscoelastic effects, quick retraction of the stamp creates a strong adhesion on top of the device, causing the anchors to break and devices to be released (Figure 2b). Slow retraction of the stamp result in the release of the devices to the new flexible substrate (Figure 2c,d). Figure 3a) illustrates a fabricated flexible array under bending with two devices switched on. Figure $3 \mathrm{~b}$ ) is a representative array of $16 \times 16$ elements before bonding. Devices show emission under forward bias at $4 \mathrm{~V}$. The electroluminescence is centered at $480 \mathrm{~nm}$ and is composed of several Fabry-Perot peaks due to the high refractive index contrast between the GaNbased epilayer and air $[5,6]$. Full device characteristics will be reported.

Conclusions: In this work, the transport of arrays of ultrathin III-nitride LEDs grown on Si (111) substrates to flexible PET/ITO substrates was demonstrated. The assembly of the devices was realized with a nano-positioning system with an in-plane accuracy of $\pm 25 \mathrm{~nm}$ in principle and specific tip and tilt manipulation down to $2.5 \times 10^{-4}$ degree.
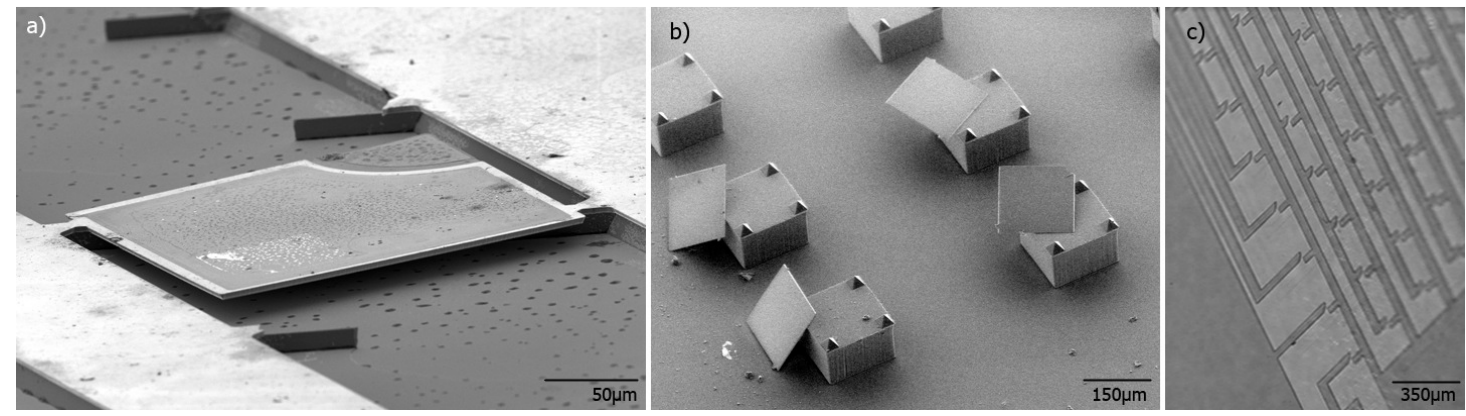

Figure 1 - SEM images of a) suspended InGaN LED, b) PDMS elastomeric stamps with LEDs, c) interconnecting ITO circuit.



c)

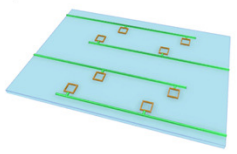

b)

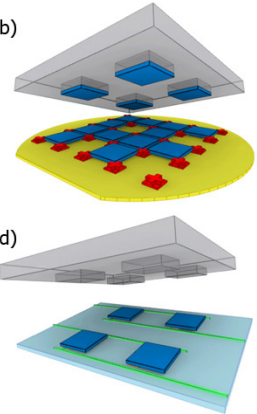

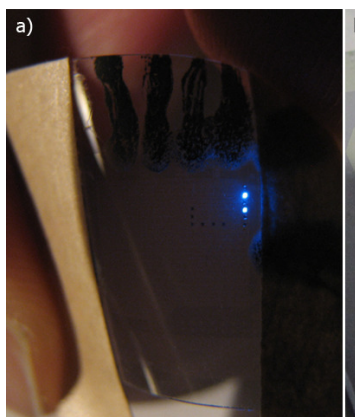

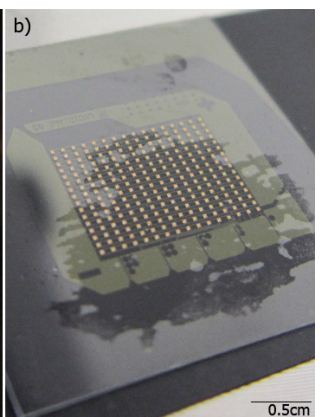

Figure 2 - Transfer printing process steps: a) device array on a donor wafer, b) elastomeric stamp for device transport, c) acceptor substrate with patterned electrodes, d) device release from stamp to

Figure 3 - Final device arrays: a) two powered devices under operation at $4 \mathrm{~V}$, b) full-array of devices (16x16) before bonding. flexible substrate.

\section{References}

[1] Dae-Hyeong Kim et al, NPG Asia Materials 4, e15, (2012)

[2] McKendry et al, IEEE Photonics Technology Letters, Vol. 21, No.12, (2009)

[3] Jong-Hyun Ahn, et al,Science 314, 1754 (2006)

[4] Bower, C.A. et al, Electronic Components and Technology Conference (ECTC), 2010 Proceedings 60th, (2010)

[5] D. Zhu et al, Proc. of SPIE 7231, 723118 (2009)

[6] D. Zhu et al, J. Appl. Phys. Lett., 109, 014502 (2011)

Acknowledments: The authors would like to thank Dr. Paul Edwards - Strathclyde Physics - for the SEM imaging. 